\title{
Production of Biosensor Controls on the Basis of Nanostructured Silicon*
}

\author{
T. S. Kachinskaya and O. M. Shmyryeva \\ Kiev National Technical University of Ukraine, 37, Prospect Peremohy, Kyiv, 03056, Ukraine \\ M. M. Melnichenko ${ }^{\dagger}$ and E. P. Yurevich \\ Taras Shevchenko Kiev National University, 2, Prospect Glushkova, Kyiv, 03127, Ukraine \\ M. F. Starodub \\ Palladin Institute of Biochemistry of NASU, 9, Leontovicha Street, Kyiv, 01601, Ukraine
}

(Received 9 October 2008; Accepted 8 April 2009; Published 6 June 2009)

\begin{abstract}
A new immune technique based on the nanostructured silicon and intended for the quantitative determination of some toxic substances is offered. The main principle of the proposed immune biosensor work is consisted in the measurement of the changes of the photocurrent of the photosensitive nanostructured silicon at the interaction of its surface preliminary treated by the specific antibodies with the toxin solution. The sensitivity of such biosensor allows determining T-2 mycotoxin at the concentration of $10 \mathrm{ng} / \mathrm{ml}$ during several minutes. Overall time of the analysis of one sample including of all procedures is about $40 \mathrm{~min}$. These indexes fit with the practice demands. [DOI: 10.1380/ejssnt.2009.677]
\end{abstract}

Keywords: Biosensing and devices; Si(100); Etching; Nano-films; Biophysics

\section{INTRODUCTION}

The protection of people health from the effect of the different toxic substances is one of main task of modern technological progress. These substances are widespread as in industry and in agriculture. Modern instrumental analytical methods allow controlling toxins in environmental objects with high precision and selectivity but among them it is absent that which can fulfill analysis in the field conditions and in on-line regime. Therefore, today there is no possibility for the wide screening which is much needed for practice. It can be provided only with creation of special devices on the basis of biosensor technology. Last years, in the area of biosensor technology, researchers pay the increasing attention to nanostructured silicon as to widely accessible and cheap material. The production of such silicon is very simple and it does not demand the use of the expensive equipment. But, the most important is that under certain conditions silicon is able to have the intensive photoluminescence in wide spectrum. For today some biosensors based on the effect of silicon photoluminescence are described $[1,2]$. Biosensors with the nanostructured silicon can be used for straight detection of the specific biological pollutants such as protein components, polysaccharides and cells used in the procedure of biotechnological synthesis. Such devices are particularly useful in the direct detection of biomolecular interactions.

In this article the technique of measurement of concentration of toxic substances by an immune biosensor on the basis of the nanostructured silicon is offered. In order to develop such sensor the first step was to achieve immobilization of the binding of biological molecules onto the

\footnotetext{
* This paper was presented at International Symposium on Surface Science and Nanotechnology (ISSS-5), Waseda University, Japan, 9-13 November, 2008.

†Corresponding author: realcrystallab@univ.kiev.ua
}

working surface.

\section{EXPERIMENTAL}

We used boron doped single-crystal silicon square wafers with resistivity of $1 \mathrm{Ohm} \cdot \mathrm{cm}$, with area of $100 \mathrm{~cm}^{2}$ and thickness of $0.3 \mu \mathrm{m}$. The surface of the wafers was not polished. Nanostructured silicon layers were prepared by stain etching in $\mathrm{HF}: \mathrm{HNO}_{3}$ solution at the room temperature, natural day-time illumination and time duration from 1 to $20 \mathrm{~min}$. Thickness of a layer nanostructured silicon changed from 3 up to $60 \mathrm{~nm}$, was supervised by parameters of technological process at chemical modification of a surface of single-crystal silicon and defined with the help of Auger electronic spectroscopy. Thickness of layers was defined as product of speed of layerby-layer argon ion etching $(3 \mathrm{~nm} / \mathrm{min})$ and time of etching [3]. Detailed investigation of chemical composition of the nanostructured silicon surface was carried out by the method of Auger electronic spectroscopy (AES) at the LAS-2000 installation intended for the surface analysis. During formation of the nanostructured silicon layer on a surface, both contents, and structure of distribution of oxygen $(\mathrm{O})$, carbon $(\mathrm{R})$ and oxidized silicon $\left(\mathrm{SiO}_{x}\right)$ change (Fig. 1). The structure of nanostructured silicon surface was studied using scanning tunnel microscope (STM) and scanning electron microscope (SEM). The morphology of the surface of the nanostructured silicon has an ordered structure and repeats exactingly the morphology of the surface of single-crystal silicon substrates, forming pores on every single relief piece (Fig. 2). We used antibodies (Ab) as ligands. In addition, antibodies with the different specificities are almost identical in their physico-chemical properties. Thus it could expect that different Ab will behave similarly. At first we studied whether Ab can be bound directly to the nanostructured silicon surface. Direct physical adsorption was choused because this binding method is simple and may provide the preservation of the biological activity of biomolecules. To detect and measure 


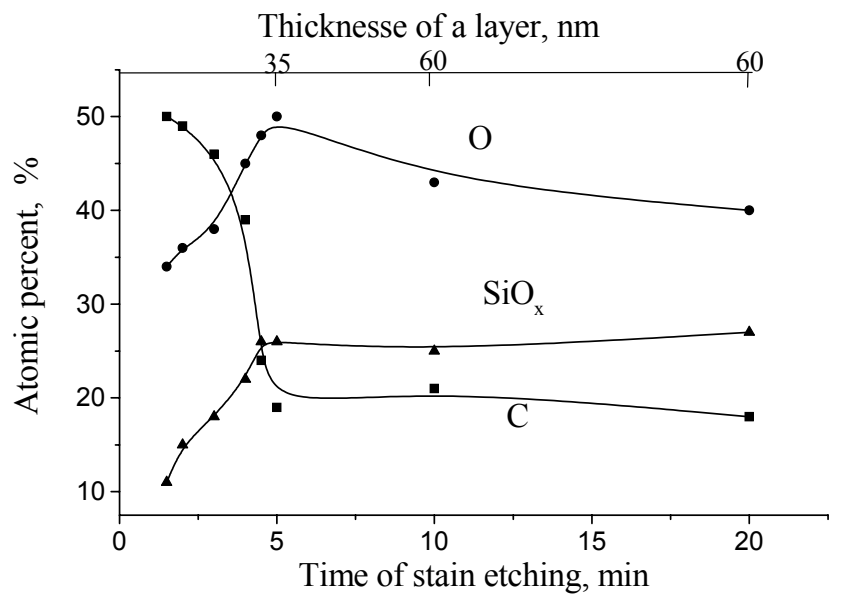

FIG. 1: Dependence of concentration $\mathrm{O}, \mathrm{C}$ and $\mathrm{SiO}_{x}$ from time of stain etching.

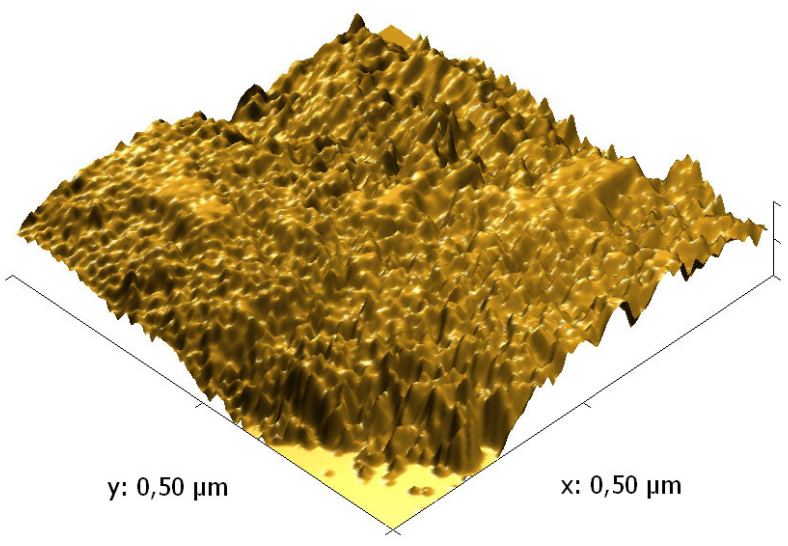

FIG. 2: STM image of the nanostructured silicon surface.

the quantity of $\mathrm{Ab}$ bound to the sensor surface we investigated the changes in the photosensitivity of the nanostructured silicon by measuring the photocurrent of photoresistor (Fig. 3). At the formation of the immune complex it is observed the small changes of the photocurrent in the dark regime. At the irradiation the photocurrent has increased in 2-5 times in dependence of the specificity of the immune complex formed. In really the main processes in the proposed biosensor are connected with the processes of the light excitation, electron generation and with subsequent electronic effects on the active surface of the nanostructured silicon. Contacts were produced with the help of a method of magnetron dispersion using of a metal mask.

\section{RESULTS AND DISCUSSION}

It was specified that solution of swine anti-human $\mathrm{Ab}$ (SwAHu from "SEVAC Praha") in tris-HCl buffer (pH 7.3) at the concentration of $5 \mu \mathrm{g} / \mathrm{ml}$ directly binds with surface of the nanostructured silicon forming specific detector element. The measurements of the photocurrent at 5 volts have shown that dark current of the biosen-

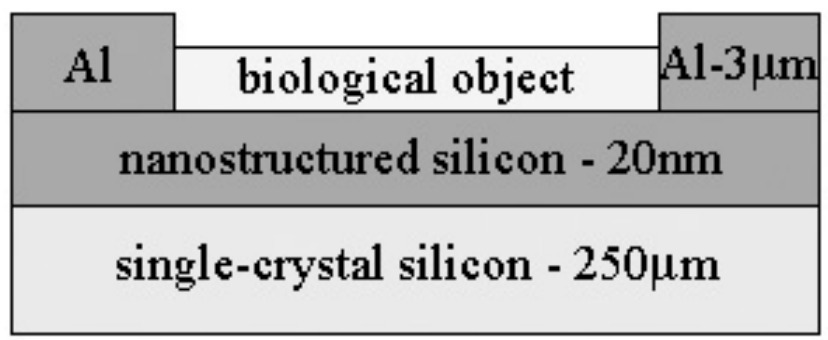

FIG. 3: Schematic scene of the photo resistor design on base of the nanostructured silicon.

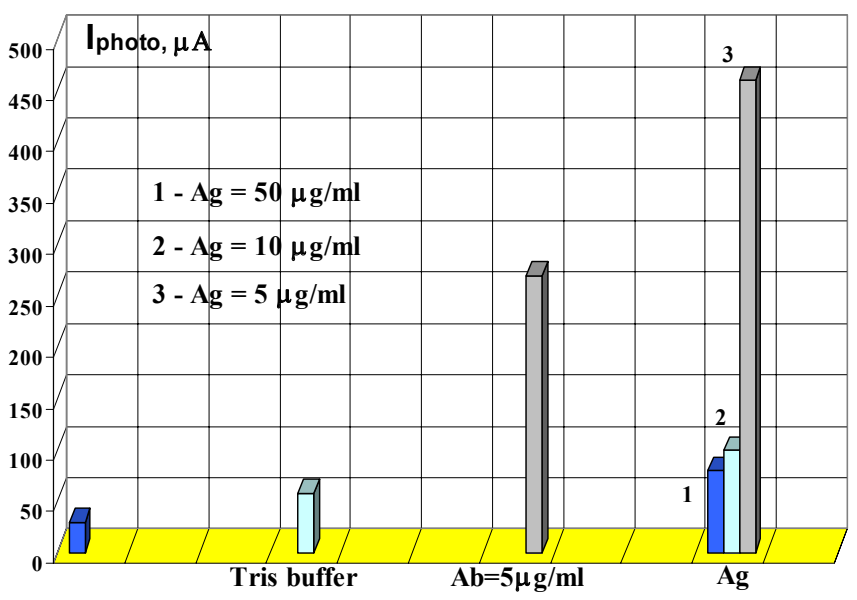

FIG. 4: Changes of the value of the photocurrent of the photoresistor before and after bounding: tris-HCl buffer ( $\mathrm{pH}$ 7.3), $\mathrm{Ab}$ and $\mathrm{Ag}$ solutions in this buffer.

sor practically does not change, but photocurrent after $\mathrm{Ab}$ binding increased almost in two times (Fig. 4). Further on the photoresistor surface with preliminary immobilized $\mathrm{Ab}$ the solution of the specific antigen $(\mathrm{Ag})$ in form of the standard human serum (from "SEVAC-Praha") at the different concentration was placed. During surface drying on air the immune complex formed. It was confirmed by the change of the photocurrent of the biosensor (Fig. 4). We have shown that $\mathrm{Ab}$ can be bound onto the nanostructured silicon surface and it is the first step towards the development of biosensors. The results indicate that $\mathrm{Ab}$ binding to the surface should facilitate the future use of the well-characterized $\mathrm{Ag}-\mathrm{Ab}$ interactions as a model. These results open the way for the application of the nanostructured silicon at the manufacture of biosensors, with the potential advantage at the control of the concentration of different toxins in real time by the application of specific antibodies.

It is well known that the semi-lethal dose $\left(\mathrm{LD}_{50}\right)$ of $\mathrm{T}-2$ micotoxin for most animals is $5 \mu \mathrm{g} / \mathrm{kg}$ of the body weight though the essential changes in the biochemical factors of the brain exist already at the dose which is equal $0.1 \mu \mathrm{g} / \mathrm{kg}[4-6]$. The above mentioned toxicity level and permissible concentration of T-2 micotoxin should be taken into account at the development of immune biosensor to be orientated on its sensitivity. Different types of $\mathrm{Ab}$ (specific and nonspecific) were immobilized on the nanostructured silicon surface. We used specific rabbit 


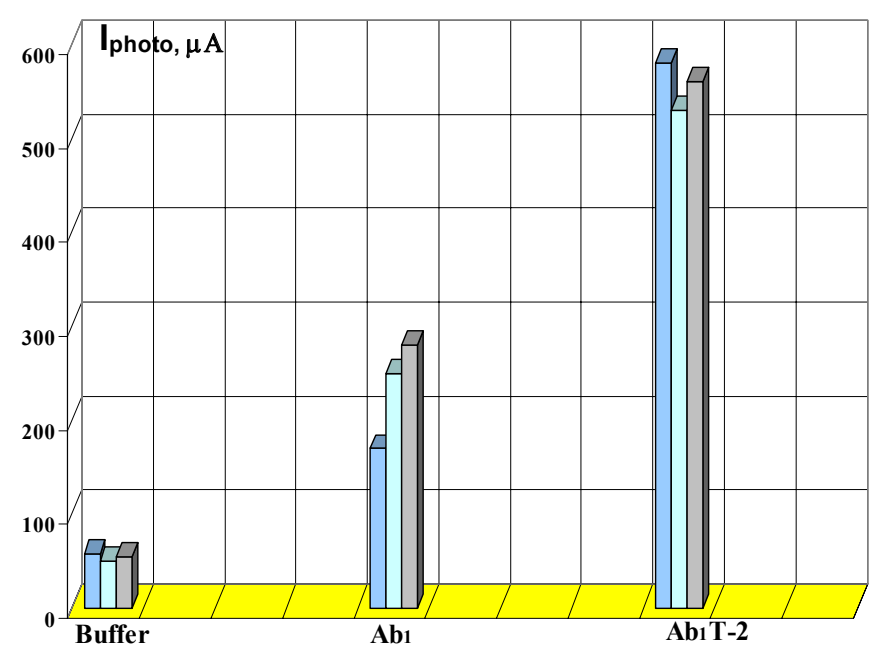

FIG. 5: Changes of the photocurrent of the photoresistor after loading of buffer, $\mathrm{Ab}_{1}$ and formation of $\mathrm{Ab}_{1} \mathrm{~T}-2$ mycotoxin complex.

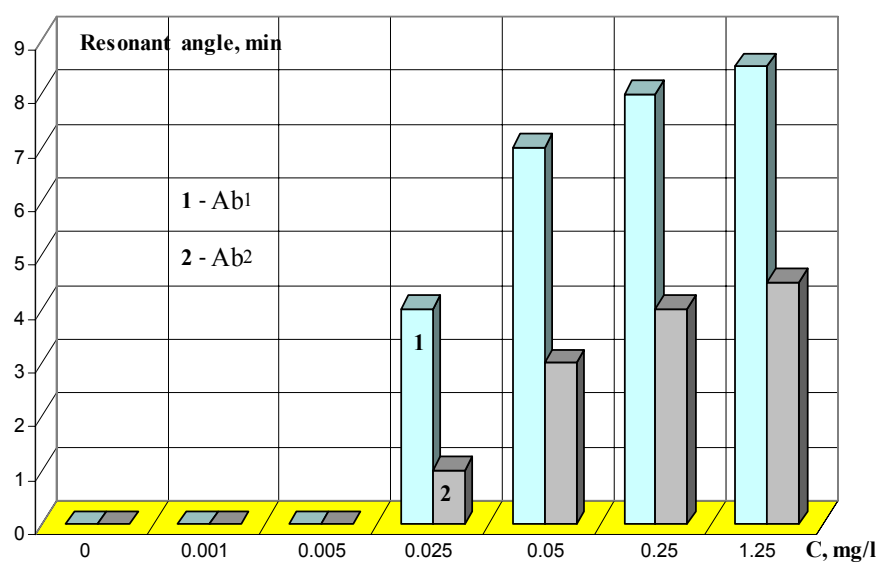

FIG. 6: Calibration curves for SPR based immune biosensor in case of application of $\mathrm{Ab} 1$ and $\mathrm{Ab}_{2}$ at the determination of T-2 mycotoxin.

Ab obtained to conjugate $\mathrm{T}-2$ with bovine serum albumin $\left(A b_{1}\right)$ and hemocyanin $\left(\mathrm{Ab}_{2}\right)$. In control experiments we applied the bovine $\mathrm{Ab}$ to retrovirus of leucosis $\left(\mathrm{Ab}_{3}\right)$ and rabbit anti-aflatoxin $\mathrm{Ab}\left(\mathrm{Ab}_{4}\right)$. All $\mathrm{Ab}$ were prepared in tris-HCl buffer, $\mathrm{pH} 7.3$, at the concentration of $10 \mathrm{ng} / \mathrm{ml}$. After Ab immobilization and photoresistor surface drying on air at a room temperature the solution of T-2 mycotoxin was downloaded above. The photoresistor surface was dried again. There is necessary to mention that T-2 mycotoxin was dissolved in the mixture of methanolwater at the ration 1:4. The concentration of toxin was 10 $\mathrm{ng} / \mathrm{ml}$. The obtained results have shown that the greatest changes of photosensitivity of the nanostructured silicon were revealed at the analysis of T-2 mycotoxin with the application of $\mathrm{Ab}_{1}$ (Fig. 5). The total duration of the fulfillment of all processes including $\mathrm{Ab}$ immobilization and steps of measurements was about $40 \mathrm{~min}$. This time may be shortened if $\mathrm{Ab}$ will be immobilized preliminary and analysis will be started beginning with the Ag loading on the photoresistor surface. The photocurrent has increased after adding the $\mathrm{Ab}$ approximately in two times. In case of immobilized $\mathrm{Ab}_{2}$ application the changes of photocurrent after their interaction with T-2 mycotoxin has increased. These changes were much less then it was found for $A b_{1}$. We suppose that it is connected with the differences in the affinity of both Ab to T-2 mycotoxin. This assumption is confirmed by the experiments with the traditional ELISA-method and the immune biosensor based on surface plasmon resonance (SPR) according to which specific signals much less for $\mathrm{Ab}_{2}$ than $\mathrm{Ab}_{1}$ (Fig. 6). The comparison of the results obtained by the proposed immune biosensor with that based on SPR (see Figs. 5 and 6) there is necessary to underline that first one is more sensitive in comparison with second one. Of course, that SPR based immune biosensor may provide much more sensitivity if the analysis will be fulfilled not "direct" way and by "competitive" of "to saturation" ways [7]. Unfortunately both last ways are more complicate in comparison with the analysis by "direct" way. The original situation was observed when we used non specified Ab, in particular $\mathrm{Ab}_{3}$ and $\mathrm{Ab}_{4}$. Both $\mathrm{Ab}$ did not stipulate changing of the photocurrent of the photoresistor. Thus, sensitivity of the proposed immune biosensor increased among used $\mathrm{Ab}$ : from $\mathrm{Ab}_{2}$ to $A \mathrm{~b}_{1}$ and any signal was not registered in case of application of non-specific $\mathrm{Ab}$, in particular, $\mathrm{Ab}_{3}$ and $\mathrm{Ab}_{4}$. The level of sensitivity to $\mathrm{T}-2$ mycotoxin is equal to $10 \mathrm{ng} / \mathrm{ml}$ that is much less than permissible pollution [4-6]. Taken into the consideration of the simplicity of the silicon production and the rapidity of the measurement fulfillment there is all reasons to conclude that the proposed immune biosensor may provide strong modern practice demands.

\section{CONCLUSION}

So, using different specific Ab to T-2 mycotoxin, by means of a new immune biosensor on the basis of the nanostructured silicon we have registered the formation of the immune complexes in real time and quickly enough (no more than $40 \mathrm{~min}$ ). The traditionally used ELISAmethod demands up to 6-8 hours for the analysis [7]. SPR based immune biosensor, early developed by us [8], allowed to fulfill analysis during 10-20 min only but its sensitivity at the "direct" way analysis was much less in comparison with the proposed immune biosensor based on the nanostructure silicon (Fig. 6). It is necessary to underline that immune biosensor based on total reflection ellipsometry has much more sensitivity but it is more complicate in respect to analysis fulfillment [9]. The described immune biosensor based on the nanostructured silicon provides very simple and faster analysis (about 10 min if photoresistor with the appropriate Ab will be preliminary prepared). The obtained calibration curves with the model solution of T-2 mycotoxin open perspective for the practical application of the proposed immune biosensor in case of the determination of others micotoxins and also others types of toxic substances with the use of their specific Ab. 
[1] N. F. Starodub, L. L. Fedorenko, V. M. Starodub, S. P. Dikij, and S. V. Svechnicov, Sensor and Actuators B 35, 44 (1996).

[2] V. M. Starodub, L. L. Fedorenko, A. P. Sisetskiy, and N. F. Starodub, Sensor and Actuators B 58, 409 (1999).

[3] A. I. Luchenko, M. M. Melnichenko, and K. V. Svezhentsova, J. Optoelectron. Adv. Mater. 9, 1431 (2007).

[4] R. W. Jr. Wannemacher, D. L. Bunner, and H. A. Neufeld, In Mycotoxins and animal foods, Eds. J. E. Smith, R. S. Henderson, (Boca Raton, Fla: CRC Press., 1991), p. 499.

[5] S. Hussein, J. Hussein, and M. Brasel, Toxicology 167, 101 (2001).

[6] L. N. Pylipenko, A. V. Egorova, I. V. Pylipenko, N. F.
Starodub, N. I. Kanjuk, and S. P. Ivashkevich, Food Sci. Technol. 1, 35 (2007).

[7] V. A. Tutel'jan, K. S. Eller, and V. S. Sobolev, Methodical recommendation for the identification and determination of T-2 toxin content in foods and foodstuff, Ministry of the public health USSR, M., 1 (1984).

[8] N. F. Starodub, L. N. Pylipeko, I. V. Pylipenko, and A. V. Egorova, Timisoara Medical J. 58, 1583 (2008).

[9] A. V. Nabok, A. Tsargorodskaya, A. Holloway, N. F. Starodub, and O. Gojster, Biosensors and Bioelectronics 22, 885 (2007). 